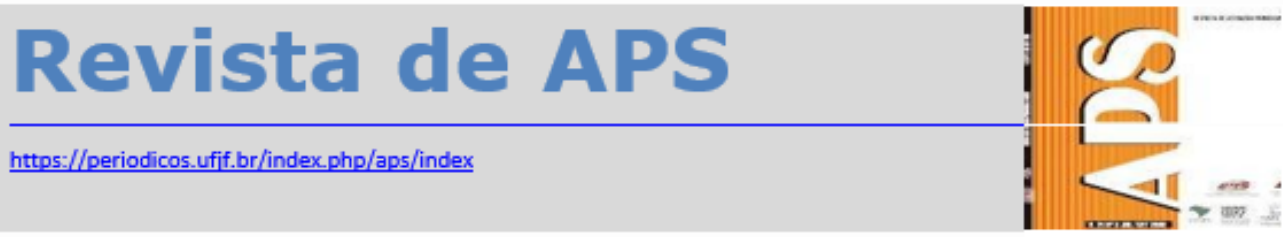

\title{
Atendimento à população masculina na atenção primária de Maracanaú-CE: estudo documental
}

\section{Assistance to the male population in the primary care of Maracanaú-CE: a documentary study}

\author{
Monalisa Chaves Magalhães ${ }^{1}$, Elza Maria da Conceição Souza ${ }^{2}$, Sara Taciana Firmino \\ Bezerra $^{3}$, Riksberg Leite Cabral ${ }^{4}$, Karla Corrêa Lima Miranda ${ }^{5}$, Manuela de Mendonça \\ Figueirêdo Coelho ${ }^{6}$
}

\begin{abstract}
RESUMO
Introdução: O presente estudo objetiva analisar os atendimentos de enfermagem destinados aos homens na Estratégia Saúde da Família (ESF), confrontando-os com as diretrizes propostas na Política Nacional de Atenção Integral à Saúde do Homem. Estudo descritivo exploratório, do tipo análise documental, de caráter retrospectivo, realizado em uma Unidade Básica de Saúde da Família, em Maracanaú-CE. Os dados foram colhidos das fichas de produção ambulatorial diária dos enfermeiros, referentes ao período de janeiro a dezembro de 2011, constatando-se que não existe nenhum dia específico para o atendimento à população masculina, bem como a predominância de procura pelo atendimento na perspectiva curativa. Os atendimentos de enfermagem aos homens ainda se distanciam do preconizado pela Política Nacional de Atenção Integral à Saúde do Homem (PNAISH). Repensar questões que permeiam a saúde do homem pode ser propulsor de mudanças necessárias ao desencadeamento de outras ações junto aos homens, estimulando-Ihes o pertencimento ao espaço de promoção, proteção e recuperação de saúde, efetivando, assim, o proposto pelo PNAISH.
\end{abstract}

PALAVRAS-CHAVE: Enfermagem. Enfermagem em Saúde Pública. Saúde do Homem.

\footnotetext{
${ }^{1}$ Curso de Enfermagem da Faculdade Metropolitana da Grande Fortaleza.

${ }^{2}$ Curso de Enfermagem da Faculdade Metropolitana da Grande Fortaleza.

3 Docente do Curso de Graduação em Enfermagem da Faculdade Metropolitana da Grande Fortaleza e da Universidade Estadual do Ceará.

${ }^{4}$ Enfermeiro. Docente da Faculdade Metropolitana da Grande Fortaleza.

${ }^{5}$ Enfermeira. Doutora em Enfermagem, Docente da UECE, nos Cursos de Graduação e no Programa de Pós-

Graduação em Cuidados Clínicos em Enfermagem e Saúde.

${ }^{6}$ Enfermeira. Mestre em Cuidados Clínicos em Enfermagem e Saúde. Docente do Curso de Graduação em

Enfermagem da Faculdade Metropolitana da Grande Fortaleza, Fortaleza. E-mail: manumfc2003@yahoo.com.br.
} 


\begin{abstract}
Descriptive exploratory study of documentary analysis type, with retrospective character, conducted in a Basic Family Health Unit in Maracanaú-CE, which aimed to analyze the nursing care for men in the Family Health Strategy, confronting them with the guidelines proposed in the National Policy for Integral Attention to Men's Health (PNAISH). The data were collected from the nurses' daily production records referring to the period from January to December 2011, which showed that there is no specific day for the care of the male population, and that the predominance of demand for care prevails in the curative perspective. Primary nursing care for men still distances itself from that recommended by the PNAISH. Rethinking issues that permeate the men's health can be propelling for changes that are necessary to trigger other actions alongside men stimulating in them the belonging to the area of promotion, protection and recovery of health, doing this way what was proposed by PNAISH.
\end{abstract}

KEYWORDS: Nursing. Nursing in Public Health. Men's health.

\title{
INTRODUÇÃO
}

A saúde do homem, nos últimos anos, tem sido um assunto cada vez mais discutido na perspectiva da saúde coletiva e, em especial, na atenção primária.

Estudos têm apontado a baixa adesão da população masculina aos serviços de Atenção Primária em Saúde. Uma das prováveis consequências dessa conduta pode estar retratada em recentes indicadores de mortalidade, cujos resultados evidenciam que, em todas as faixas etárias e em decorrência de quase todas as causas, os homens morrem mais precocemente do que as mulheres. ${ }^{1}$

A Atenção primária em Saúde considera o ser humano em diversas perspectivas, desde a integridade biológica, até os aspectos psicossociais para o direcionamento da promoção da saúde, considerando suas singularidades e complexidades como base para a redução dos adoecimentos que possam comprometer suas possibilidades de um viver saudável. ${ }^{2}$ Dessa forma, o desejável seria que ações de cuidado à população masculina, dentro dos serviços de saúde, ocorressem de forma equitativa com os demais segmentos populacionais.

Um ponto importante a se pensar é a questão de gênero na sociedade ocidental, na qual o homem apresenta-se como o sexo forte, pilar estruturante da família, assumindo, assim, riscos que interferem em suas condições de saúde. Tal estereótipo também define a forma como os homens usam e percebem os seus corpos. $\mathrm{Na}$ perspectiva de seus modelos de masculinidade, o homem, muitas vezes, assume 
comportamentos considerados pouco saudáveis, que estão relacionados a um modelo de masculinidade idealizada, a masculinidade hegemônica. ${ }^{1}$

Nos modelos afirmados pela sociedade, o ser masculino é invulnerável, forte, e o seu adoecimento apresenta-se como demonstração de fraqueza, permitida apenas ao sexo feminino. A percepção de invulnerabilidade e do modelo hegemônico de masculinidade faz com que o homem desvalorize o autocuidado com a saúde, colocando-a em segundo plano. ${ }^{3}$ Tal arquétipo aponta os cuidados de saúde como algo inerente à mulher. ${ }^{4}$

Além dos homens terem dificuldade em reconhecer suas necessidades, cultivando o pensamento mágico que rejeita a possibilidade de adoecer, os serviços e as estratégias de comunicação privilegiam ações de saúde à criança, ao adolescente, à mulher e ao idoso. Os serviços de saúde ainda devem ser reestruturados para o atendimento dessa demanda, não apenas física, mas uma modificação, principalmente qualitativa, no que se refere aos profissionais e à própria comunidade. ${ }^{5}$

Os agravos decorrentes desta conjuntura conduzem a problemas de saúde pública, pois o acesso dos homens aos serviços de saúde apresenta-se em predominância na assistência especializada. A resistência masculina às práticas preventivas somada à fragilidade de programas de saúde pública, com foco sobre este segmento, apresenta pesada influência financeira à sociedade, bem como abalos à estrutura familiar, tendo em vista que algumas condições de saúde são desestruturantes às pessoas de seu convívio. ${ }^{6,4}$

A captação da população masculina para a atenção primária deve ser realizada pela equipe da Estratégia Saúde da Família (ESF), composta, dentre outros profissionais, pelo enfermeiro. A partir da identificação das necessidades do homem e de seus problemas, estratégias devem ser pensadas para facilitar a sua adesão às ações preventivas ofertadas pelo serviço.

O enfermeiro é um profissional atuante e desempenha papel fundamental na ESF. Diversas ações podem ser instituídas pelos profissionais enfermeiros na promoção da saúde do homem, tanto assistenciais, como educativas e preventivas: consultas individuais, aferição de pressão arterial, grupos educativos, imunização, entre outros . ${ }^{7}$

A Política Nacional de Atenção Integral à Saúde do Homem (PNAISH) foi criada, em 2008, pelo Ministério da Saúde (MS), na tentativa de fomentar a saúde como direito 
básico do homem brasileiro. Tal política é direcionada aos homens, na faixa etária de 25 a 59 anos, grupo este que corresponde a $20 \%$ do total da população brasileira, justificando sua relevância no cenário nacional. Ademais, não se deve negar a consideração desta parcela significar importante contingente da força produtiva brasileira. ${ }^{6}$

Nesse sentido, a PNAISH propõe a organização dos serviços de saúde e suas linhas de cuidado, para resguardar a integralidade das ações destinadas aos homens. 0 reconhecimento de que a população masculina acessa o sistema de saúde, por meio da atenção especializada prediz a necessidade de avançar na priorização da atenção primária, reduzindo agravos evitáveis, bem como processos de recuperação prolongados. ${ }^{6}$

Para alcançar essa população, o MS entrelaça a PNAISH com a Política Nacional de Atenção Básica (PNAB), reforçando a importância da prevenção e promoção da saúde, no intuito de incrementar o aumento da expectativa de vida e a redução dos índices de morbimortalidade por causas preveníveis e evitáveis nessa população. ${ }^{8,6}$

Sabendo-se a importância do cuidado à população masculina, dentro das Unidades Básicas de Saúde da Família (UBASF) e considerando a PNAISH como uma política nova e ainda em desenvolvimento, considera-se relevante acompanhar a implantação e desenvolvimento dessa proposta, concebendo seus ganhos e suas possíveis deficiências como espelho para mobilização de melhorias. O presente estudo objetiva conhecer o perfil dos atendimentos de enfermagem destinados aos homens na ESF, confrontando-os com as diretrizes propostas na PNAISH.

\section{METODOLOGIA}

Estudo descritivo exploratório, do tipo análise documental, predominantemente quantitativo, de caráter retrospectivo. As fontes documentais foram analisadas sob a perspectiva histórica e social do momento em que o município vive o desenvolvimento do PNAISH e produzidas de acordo com os objetivos do estudo.

A pesquisa foi realizada em uma Unidade Básica de Saúde da Família em Maracanaú - CE, a qual possui quatro equipes da ESF. Os dados foram colhidos, a partir de fichas de produção ambulatorial diária dos enfermeiros de cada equipe, produzidas 
nos meses de janeiro a dezembro do ano de 2011. As fichas de produção ambulatorial consistem em formulário padronizado, nos quais os profissionais registram nome, sexo, idade e motivo da procura pelo atendimento do usuário, podendo constar até 15 atendimentos. Integraram essa amostra 6.320 atendimentos em 730 fichas de produção ambulatorial.

Para a coleta dos dados, utilizou-se formulário estruturado com questões relativas à idade e ao motivo do atendimento. Observou-se, também, a agenda e o cronograma de atividades dos profissionais, com o intuito de reconhecer as ações que tenham como foco principal o atendimento ao homem.

Como critério de inclusão, decidiu-se que deveriam ser captados apenas os registros de fichas de atendimento ambulatorial dos enfermeiros, que as estivessem preenchido, de forma completa e legíveis e atendimentos a homens na faixa etária de 25 a 59 anos. Ao analisarmos as fichas de produção ambulatorial, no ano de 2011, totalizaram-se 6.320 atendimentos de enfermagem, destes, $4.245(67,16 \%)$ foram destinados às mulheres, $1.172(18,54 \%)$ às crianças e 903 (14,28\%) aos homens.

Esta pesquisa faz parte de um macro projeto intitulado "Estratégia Saúde da Ffamília em Maracanaú/CE: Análise Histórica Documental e Evolução Temporal dos Indicadores de Saúde". O mesmo foi submetido ao Comitê de Ética em Pesquisa da escola de Saúde Pública do Ceará, aprovado com o parecer no 229/2011, encontrandose dentro das normas que regulamentam a pesquisa com seres humanos do Conselho Nacional de Saúde, resolução no 196, quando se assinou o termo de fiel depositário.

\section{RESULTADOS E DISCUSSÕES}

Conhecer a organização dos serviços se fez necessidade primeira. Na UBASF pesquisada, a agenda de trabalho dos enfermeiros encontra-se claramente segmentada em blocos delimitados por áreas programáticas: saúde da mulher (planejamento familiar, prevenção do câncer de colo uterino, pré-natal, puerpério), saúde da criança (imunização e puericultura) e saúde do adulto (atendimento para hipertensão arterial e diabetes mellitus, hanseníase e tuberculose).

Além dos turnos de trabalho destinados às consultas ambulatoriais aos grupos de pacientes já mencionados, também compõem esta agenda atividades, como: visita 
domiciliária, grupos educativos, atividades na escola e reunião de equipe. Porém, não se identificou em nenhum dos cronogramas propostos (estes estão afixados nos corredores da unidade de saúde) um turno claramente destinado a atendimento específico ao público masculino.

Com essa realidade, surgem duas reflexões. A primeira seria que o atendimento ao homem não ficasse enrijecido em dias determinados, possibilitando a essa clientela acesso livre e, em dias diversos, durante a semana, podendo ser um facilitador para o acesso desses sujeitos à UBASF.

Já, na segunda, indaga-se como o homem consegue ser atendido em dias, quando o cuidado está destinado a outro perfil de público, e, assim, a disposição dos turnos de atendimentos poderia ser um dificultador para o ingresso do homem nos serviços de saúde da atenção primária, diante da não identificação desses sujeitos com os serviços ofertados, sem espaço para atendimento de suas demandas.

Compreende-se que as equipes da ESF deveriam acolher as necessidades da população sem discriminação de raça, idade ou gênero, porém, deve-se reconhecer o homem como sujeito de necessidades singulares, exigindo, assim, por parte do enfermeiro, bem como de toda a equipe, atenção específica que possa abraçar suas demandas de saúde de forma integral. ${ }^{7}$

Essa questão inicial pode ser pensada, levando em consideração os dados sobre os atendimentos de enfermagem. Os atendimentos direcionados à população masculina englobam os homens de 18 a 69 anos. Foram analisados, neste estudo, os atendimentos direcionados aos homens, na faixa etária entre 25 e 59 anos, recorte preconizado pelo Ministério da Saúde (MS) como foco para o programa Saúde do Homem. Apoiados nessa faixa etária, identificaram-se 419 atendimentos, perfazendo 46,40\% das consultas direcionadas aos clientes masculinos e apenas $6,6 \%$ da demanda total de atendimentos.

Entre os 419 usuários atendidos pelas equipes da ESF, durante o ano de 2011, identificou-se o predomínio da faixa etária de 45 a 54 anos, com 163 (38,90\%) homens, seguida das faixas etárias de 55 a 59 anos, com 151 (36,03\%) homens, e 35 a 44 anos, com 59 (14,08\%) homens. A menor procura à UBASF ocorreu por homens entre 25 a 34 anos com 46 (10,97\%) usuários.

Outros estudos demonstram o predomínio de atendimentos aos homens na faixa etária de 45 a 54 anos. ${ }^{9} \mathrm{Na}$ análise dos motivos de procura pelo serviço, verificou-se o 
predomínio de atendimentos relacionados às doenças crônicas.

Tabela 1- Tipos de atendimentos realizados pelos enfermeiros a homens de 25 a 59 anos em 2011, Maracanaú - CE, 2013.

\begin{tabular}{lcc}
\hline \multicolumn{1}{c}{ Tipo de atendimento } & $\mathrm{n}$ & $\%$ \\
\hline Consulta de Enfermagem em Hipertensão Arterial Sistêmica & 198 & 47,2 \\
Consulta de Enfermagem/Triagem & 27 & 6,4 \\
Consulta de Enfermagem em Tuberculose & 60 & 14,3 \\
Consulta de Enfermagem em Diabetes Mellitus & 52 & 12,4 \\
Outros & 82 & 19,5 \\
Total & 419 & 100 \\
\hline
\end{tabular}

Fonte: elaborada pelos autores

Em relação à consulta de enfermagem, destaca-se a importância do reconhecimento de ações próprias à profissão, cuja consulta é ação privativa do enfermeiro.

Observa-se que o tratamento e acompanhamento para hipertensão arterial sistêmica (HAS) representa quase a metade da procura por atendimento de enfermagem. O inquérito destaca que a prevalência global da HAS é quase equitativa entre homens e mulheres, todavia, esta é mais elevada nos homens até os 50 anos. ${ }^{10}$ Esse dado nos leva a pensar que a predominância da faixa etária atendida de 45 a 54 anos corrobora tal dado epidemiológico, justificando o maior número de atendimentos ter sido demandado por tal patologia.

Destaca-se a importante atuação da enfermagem na atenção à saúde do portador de hipertensão, conduzindo orientações pertinentes ao uso de medicamentos, dieta, atividade física, interrupção do tabagismo, controle do etilismo, diminuição na ingesta de sal, entre outros, a fim de contribuir positivamente na habilidade de autocuidado do indivíduo e diminuição dos danos decorrentes da evolução da doença. ${ }^{11}$

Seguidos pelas consultas a pacientes com hipertensão, estão registrados atendimentos diversos que, ao serem totalizados, não quantificaram, isoladamente nem $2 \%$ da demanda de atendimento, denotando a esporadicidade de tais cuidados, sendo agrupadas em objetivos diversos, tais como: notificação para atendimento antirrábico, planejamento familiar, hanseníase, suspeita de dengue, sintomas respiratórios, 
solicitação de exames, diarreia, transtorno mental, guia de referência para atendimento especializado, sintomático dermatológico, gripe, virose e quadros álgicos diversos.

Os atendimentos categorizados como diversos destacam-se por apresentarem como características predominantes situações de adoecimento, porém observa-se tímido movimento em busca de atendimentos, possivelmente preventivos, como o planejamento familiar e a solicitação de exames. Não se pode afirmar se a solicitação desses exames foi por motivos preventivos ou diagnósticos. O estudo relata que essa reduzida procura por ações voltadas para a promoção da saúde e prevenção de doenças se dá pelo receio de os homens descobrirem algum adoecimento. ${ }^{9}$

$\mathrm{O}$ atendimento para o seguimento de tuberculose e diabetes mellitus (DM) corrobora com os achados somados aos demais dados, revelando uma realidade organizacional do serviço de saúde, que ainda se encontra focado ao atendimento ambulatorial e numa perspectiva curativa. Assim, tal conjuntura da rede básica de saúde tem exercido influência na modelagem do serviço de atenção à saúde do homem, distanciando-se das proposições preventivas da PNAB e da PNAISH.

Dos registros categorizados como consulta de enfermagem para triagem, não foi possível apreender detalhes apurados sobre o direcionamento e foco de tais momentos, uma vez que não constavam maiores informações nos registros analisados.

A triagem é o passo inicial para avaliação, seleção e encaminhamento para o atendimento adequado para cada cliente. Acredita-se, também, ser um encontro que produz diálogo, no qual o enfermeiro pode ouvir as queixas do paciente e conhecer suas necessidades, sejam elas físicas, psicológicas ou de ordem social. ${ }^{12}$

Acolher as diversas demandas, a partir do encontro entre indivíduos numa perspectiva, que transcende apenas o cuidado biológico, mas que englobe uma composição ética e afetiva é uma potente ferramenta de trabalho em saúde, mas carece de uma escuta atenta por parte do profissional na tentativa de minimizar os ruídos produzidos por uma comunicação ineficaz e fria. ${ }^{13}$

Nada foi encontrado nos mapas que sugerisse grupos educativos voltados exclusivamente à clientela masculina, bem como ações preventivas destinadas à imunização e saúde do trabalhador, embora se possa considerar que as ações grupais e educativas registradas possam ter a participação de homens. 


\section{CONCLUSÕES}

De acordo com os resultados analisados, percebeu-se que os atendimentos de enfermagem à população masculina ainda se distanciam da visão preventiva preconizada pelo PNAISH. Tal fato é prontamente identificado nos quadros que apresentam os cronogramas dos enfermeiros expostos na unidade básica, denunciando que o olhar para a saúde do homem ainda procura espaço na organização do cuidado prestado por esses profissionais na ESF.

O quantitativo reduzido de atendimentos destinados a esse grupo coaduna com diversos estudos, apontando que a perspectiva coletiva direcionada à saúde do homem ainda carece de maior apoio, investimento e desenvolvimento. Percebe-se que prevalecem ações de enfermagem, de forma mais significativa aos programas ministeriais estabelecidos, como controle de hipertensão, diabetes e tuberculose.

Não se pretende neste estudo negar a necessidade de cuidado às patologias existentes na população. Reconhece-se que o olhar para tal condição é fundamental para prevenção de complicações e mortes por causas evitáveis.

Entretanto, considera-se a necessidade de inclusão da perspectiva preventiva como propulsora de qualidade de vida. Acredita-se que a população masculina, ao inverter sua percepção em relação ao cuidado com a saúde (do curativo para o preventivo), pode romper com sua invisibilidade no tocante às necessidades de gênero e modificar o perfil de morbimortalidade dessa classe.

Quanto aos gestores em suas diversas esferas, os enfermeiros integrantes da equipe de saúde, bem como a outros profissionais, sugere-se a urgência em adotar atitude de acolhimento à comunidade masculina, reconhecendo suas necessidades relativas não só ao tempo disponível entre um dia de trabalho e outro para cuidar da saúde, mas no que diz respeito aos estereótipos cultivados ao longo dos séculos junto a esta clientela.

O movimento de repensar questões, que permeiam a saúde do homem, pode ser propulsor de mudanças profissionais e organizacionais necessárias ao desencadeamento de novo pensar junto aos homens, estimulando nestes o pertencimento ao espaço de promoção, proteção e recuperação de saúde, efetivando, assim, um dos propósitos do PNAISH. 


\section{REFERÊNCIAS}

1. Couto $M T$, et al. O homem na Atenção Primária à Saúde: discutindo a (in)visibilidade a partir da perspectiva de gênero. Interface Comun. Saúde Educ. 2010; 14(33):257-270.

2. Brasil. Ministério da Saúde. Política Nacional de Atenção Básica à Saúde. [Internet]. 2006 maio; 21(1) [Citado 2013 jun. 16]. Disponível em: <www.conass.org.br/admin>.

3. Salimena AM, Sacramento LC, Salimena AMO, Greco RM, Paschoalin HC. Saúde do homem e atenção primária: o olhar da enfermagem. Rev. APS. 2013; 16(1):50-59.

4. Figueirêdo W. Assistência à saúde dos homens: um desafio para os serviços de atenção primária. Ciênc. Saúde Coletiva. 2005; 10(1):105-9.

5. Silva PAS, et al. A saúde do homem na visão dos enfermeiros de uma unidade básica de saúde. Rev. Esc. Anna Nery (impr.). 2012; 16(3):561-568.

6. Brasil. Ministério da Saúde. Política Nacional de Atenção Integral à Saúde do Homem: princípios e diretrizes. Brasília (DF); 2008.

7. Ximenes Neto FRG, et al. Trabalho do enfermeiro na atenção à saúde do homem no território da estratégia saúde da família. Revista Eletrônica Gestão \& Saúde. 2013; 04(01):1741-1756.

8. Laurenti R, Jorge MHPM, Gotlieb SLD. Perfil epidemiológico da morbimortalidade masculina. Ciênc. Saúde Coletiva. 2005; 10(1):35-46.

9. Vieira KLD, et al. Atendimento da população masculina em unidade básica saúde da família: motivos para a (não) procura. Esc. Anna Nery (impr.). 2013; 17 (1):120 127.

10. Cesarino $C B$, et al. Prevalência e fatores sociodemográficos em hipertensos de São José do Rio Preto - SP. Arq. Bras. Cardiol. 2008; 91(1):31-35.

11. Carvalho CG. Assistência de enfermagem aos portadores de hipertensão arterial sistêmica e diabetes mellitus: educação em saúde no grupo hiperdia. e-Scientia. [Internet] 2012; 5(1):9-46 [Citado 2013 jun. 16]. Disponível em: $<$ www.unibh.br/revistas/escientia/>.

12. Azevedo JMA, Barbosa MA. Triagem em serviços de saúde: percepções dos usuários. Rev. Enferm. UERJ. 2007; 15(1):33-9.

13. Nascimento PTA, Pekelman R. Acesso e acolhimento: "ruídos" e escutas nos encontros entre trabalhadores de.

Submissão: agosto de 2013.

Aprovação: fevereiro de 2018. 